\title{
Getting more efficient Rapid Response System (RRS) utilization by the use of a general ward based deteriorating patient contract
}

\author{
Michael Buist ${ }^{*}$, Stuart Marshall², Bill Shearer ${ }^{3}$, Monica Finnigan ${ }^{3}$, Tonina Hore ${ }^{3}$, Tamica Sturgess ${ }^{3}$
} and Stuart Wilson ${ }^{3}$

\begin{abstract}
Objective: To determine if a general ward-based plan to address the deteriorating patient could improve RRS utilization over and above a mandatory organizational policy and procedure.

Design: A two stage methodology: First, engagement with ward providers and users of the RRS to generate ward-based interventions; Second, measurement of the incidence of missed/delayed RRS calls, RRS calls, cardiac arrests and unplanned intensive care unit admission both prior to the intervention and 12 months post implementation.

Setting: Monash Health is a comprehensive healthcare network with 570 adult in-patient beds across four metropolitan teaching hospitals in the south-eastern sector of Melbourne.

Results: The interventions selected for intervention were: (1) a ward based agreement on how to best locally optimize management of the deteriorating patient,(2) an improved communication protocol, and (3) a revision of the education program for staff. Post intervention the aggregate rate of missed/delayed RRS calls reduced across all wards (1.05 to 0.34 per 1000 bed days, $p=0.049$ ). This occurred without a change in the rate of RRS activation (7.98 per 1000 bed days pre-intervention versus 7.85 bed days post-intervention). The incidence of cardiac arrests or unplanned interventions did not change post intervention.
\end{abstract}

Conclusions: Engagement of the users of a system like the RRS activation protocol can improve compliance rates with protocols when the users have ownership of the process.

Keywords: Rapid Response Systems (RRS), Medical Emergency Team (MET), Patient safety, Cardiac arrest, Hospital culture

\section{Background}

Regardless of the debate over the efficacy of the RRS, they have been widely adopted [1]. However for the RRS to be effective it needs to be utilized appropriately $[2,3]$. In 2007 the Quality and Safety unit in our organization identified eleven sentinel cases where significant patient harm occurred in association with the failure of bedside staff to activate the RRS. Attempts to rationalize and pattern match the underlying features of our incidents using clinical reviews and root cause analyses were unsuccessful. There appeared to be no obvious cause in terms of poor staff training, lack of motivation or

\footnotetext{
* Correspondence: Michael.Buist@utas.edu.au

${ }^{1}$ The Centre for health Services Research, School of Medicine University of Tasmania, Private Bag 3513, Burnie, Tasmania 7320, Australia

Full list of author information is available at the end of the article
}

understanding of the patients' clinical states; the usual factors noted in the literature [4-9].

To better understand this problem, we previously undertook a study to determine the incidence and factors behind the phenomenon of not activating the RRS $[10,11]$. In the point prevalence part of this study [10], undertaken over a 24 hour period, we found an incidence of failure to activate the RRS of $42 \%$. The reasons we found for failure to activate the RRS were predominantly socio-cultural factors amongst the bedside ward clinical staff; namely a sense that the clinical situation was under control, that the critical care team had already reviewed the patient, and that the bedside treating team had enough experience to manage the situation. We concluded that the failure to activate the RRS was not because of failure to appropriately recognize 
and manage a deteriorating patient, but rather there was a face validity problem with activation of the RRS. For example, in this study [10] there was only one Intensive Care Unit (ICU) admission from all the patients who did not appropriately get a RRS response. The remainder of the patients did not need any significant clinical intervention. However, this one patient needed prolonged multi-organ support and several reoperations. What we found is that ward clinical staff interpret the RRS activation criteria against the clinical context and their own clinical ward or unit socio cultural practice beliefs. From that study we could see that most of the time the ward clinical staff were correct in their assumptions about the trajectory of the patient outcomes. On the one occasion that they erred there was significant cost to both the patient and the organization. In essence, both the sensitivity and specificity of commonly utilized RRS activation criteria are too low to significantly change ward staff beliefs about the RRS [12-15].

To improve the management and outcome of deteriorating patients in our organization we decided to try a different approach to our traditional top down policy and procedure for mandatory RRS activation. Instead, we developed a socio-cultural intervention with the staff in the general wards. The aim of this study was to determine the effectiveness of that intervention to improve the utilization of RRS to prevent such sentinel events. In essence what we wanted was the right patient getting the RRS response and also not having the RRS attend calls on patients where no interventions were required. To do this we wanted to blend the organization's mandatory RRS activation policy with some of those unwritten ward socio cultural beliefs in an effort to get the right response for each patient.

\section{Methods}

\section{Ethics approval}

The Monash Health research and ethics committee exempted this study from requiring ethics approval as it fulfilled the NHMRC criteria for a quality assurance project.

\section{Setting}

Monash Health is a comprehensive university tertiary health care provider for the south-eastern suburbs of Melbourne. Over 13,000 staff service a catchment population of 880,000 and provide acute health services at four hospital sites. All acute services are provided with the exception of burns, spinal injury, and major organ transplantation. Monash Health was an early and enthusiastic adopter of the RRS with establishment of the Medical Emergency Team (MET) in one of our hospitals in 1996. This resulted in substantial and significant improvements in patient outcome within our hospitals
$[16,17]$ and this system of care was standardized throughout the organization by 2006. Additionally our organization committed to training all junior members of clinical staff and MET responders in the purpose built simulation centre [18].

\section{Intervention wards}

Six sample wards were selected (Table 1) that represented a mix of surgical and medical patients, and wards where from our previous study the incidence of end points (cardiac arrest, ICU/HDU admission, and unexpected death) were in the middle range $[10,11]$.

\section{Study design}

There were two parts to this study. (a) First, a process of engagement of actual users of the RRS to select interventions that may improve utilisation of the RRS. (b) Second, testing of these interventions in the selected wards .

\section{(a) Selection of interventions}

Twenty-four participants were selected who represented users of the RRS. These included junior and senior clinicians who may activate the RRS in the clinical areas and staff attending as part of the RRS such as ICU nurses. These participants attended a one day workshop with the aim of selecting up to three interventions to trial in the six study wards. This selection process was facilitated by the presentation of results from our previous work that documented failure of RRS activation. To assist with the selection of the interventions, participants were asked to focus on four issues with the current RRS.

Current RRS issues/questions 1 . How can RRS calls be sensitive to all the different clinical areas? 2. How can we prevent prolonged delays in RRS activation? 3. How can we make RRS activation criteria more visible to staff? 4. How can we make sure that the treating (parent) clinical team continue to have responsibility for the patient.

From the socio-cultural intervention workshop day, the 24 users of the RRS put 60 proposals forward about how the process could be improved. Suggestions that were duplicated or clearly unworkable due to extreme cost were excluded. The authors then rated the remaining 14 proposals for 5 criteria (effectiveness, acceptability, feasibility, cost and sustainability) using a score of 1 to 5 for each criteria in each proposal. After this, a further two proposals were excluded. The first being the use of colour coded charts on the basis of proposed national implementation by the Australian Commission on Quality and Safety [19]. The second was the simulation-based education program for nurses and doctors about the RRS in our organization as this was a suggestion that had been implemented already [18]. At the conclusion of this process, three proposals adopted for intervention in the six wards: 
Table 1 Clinical and Demographic characteristics of the pilot wards $(n=6)$

\begin{tabular}{llllll}
\hline Ward & Hospital & Surgical or Medical & $\begin{array}{l}\text { Elective or } \\
\text { Acute } \\
\text { admissions }\end{array}$ & $\begin{array}{l}\text { Number of } \\
\text { beds }\end{array}$ & $\begin{array}{c}\text { Average occupancy } \\
\end{array}$ \\
& Monash Medical Centre & Surgery (Renal and Vascular) & Both & 26 & $91 \%$ \\
\hline $1(34 \mathrm{~N})$ & Monash Medical Centre & Medical (General Medicine) & Acute & 28 & $92 \%$ \\
$2(44 \mathrm{~S})$ & Casey Hospital & Both (General Medicine and Surgery) & Both & 27 & $54 \%$ \\
$3($ Ward A) & Dandenong Hospital & Both (Gastroenterology and general surgery) & Acute & 28 & $96 \%$ \\
$4($ Surgical Unit 1) & Dandenong Hospital & Medical (Respiratory Medicine) & Both & 36 & $99 \%$ \\
$5($ West 4) & Moorabbin Hospital & Surgery (ENT, Urology and Orthopaedics & Elective & 25 & $74 \%$ \\
6 (Ward 3) & & &
\end{tabular}

(1) Individual ward based agreements for the activation of the RRS (Figure 1). The idea of individual agreements was born out of the realization that health professionals in specialized clinical areas often considered themselves experts in a particular mode of deterioration. For example, an abnormal heart rate may be more appropriately managed in an area of predominantly cardiology patients than on a neurology ward. The staff on each unit were given clear boundaries of clinical freedom to work within, depending on their individualized agreement. Agreements differed slightly in the timeframes for response and the individuals to whom the clinical reviews were escalated.

(2) Clear communication protocols were introduced about how the paging system should be used. These ensured details were given about the patient's identity, what was required and how urgent the request was. The model used was based on the ISBAR (Identify, Situation, Background, Assessment, Request) situational briefing tool $[20,21]$.

(3) A revision of the education process for new staff occurred in parallel with these other two interventions. An inter-professional induction program was developed that specifically addressed the care of the deteriorating patient [22].

\section{(b) Intervention testing on the six wards}

This was undertaken using a before and after analysis of the incidence of all RRS activations, missed/delayed RRS activations, and cardiac arrest calls on each of the six wards. The primary endpoint for this study was the incidence of missed/delayed RRS activations. The definition of a missed/delayed RRS activation was that the patient, in the 24 hours prior to either a RRS activation, cardiac arrest call, or admission to the critical care unit, fulfilled the RRS activation criteria and that the activation did not occur at that time. The activation criteria are those that we have previously published [16].The secondary endpoints were the incidence of RRS activations and cardiac arrest calls.
Post implementation data was collected for two periods, initially after implementation and at one year. For each period, the incidence of the various endpoints was determined over a 10 week period, simultaneously across the six intervention wards by daily examination of the cardiac arrest, and RRS team activation databases and review of the previous 24 hour's admissions to the critical care unit. The number of bed days for each ward was obtained for each ward from a centralized database. The second post observation period after one year was undertaken to allow for adequate training and socialization of the interventions. Minor changes were also made to the cognitive aid that was provided with the clinical observation sheet to help the clinical staff implement the changes. To prevent confusion, the results of the immediate post intervention phase have been omitted.

\section{Statistical analysis}

Statistical analyses were performed using SPSS statistics version 20 (IBM). Pre- and Post-intervention data on the rates of RRS activations, missed RRS activations, cardiac arrest calls and unplanned Intensive Care Unit (ICU) admissions were compared using binary logistic regressions. A level of $\mathrm{p}<0.05$ was taken as statistically significant.

\section{Results}

The interventions selected for implementation were: a ward based agreement on how to best locally optimize management of the deteriorating patient; an improved communication protocol; and a revision of the education program for staff. Post intervention the aggregate rate of missed/delayed RRS calls reduced across all wards ( 1.05 to 0.34 per 1000 bed days, $\mathrm{p}=0.049$ ). This occurred without a change in the rate of RRS activation (7.98 per 1000 bed days pre-intervention versus 7.85 bed days post-intervention). The incidence of cardiac arrests or unplanned interventions did not change post intervention (Table 2). 


\section{North Abnormal Vital Signs Response Sheet}

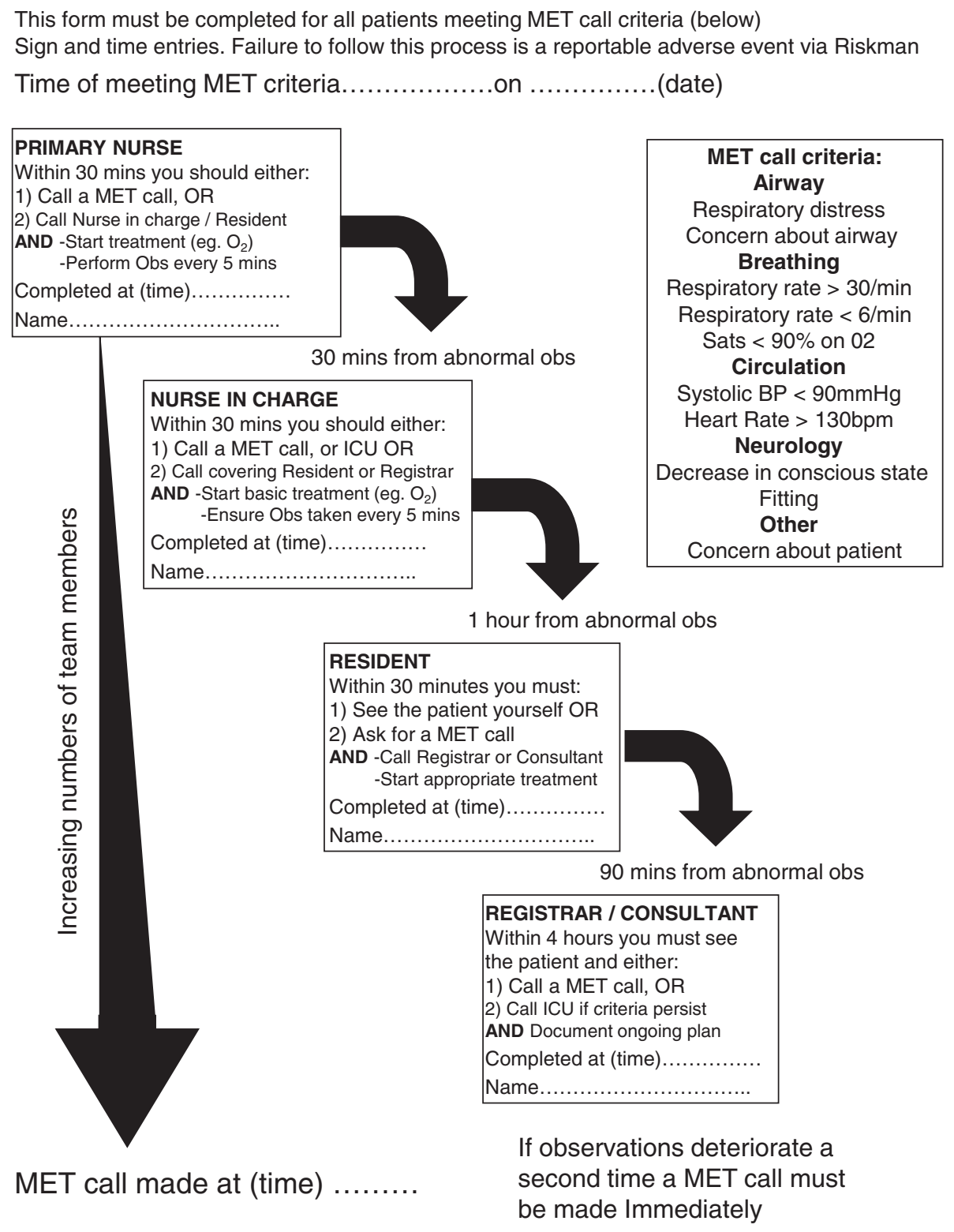

Figure 1

\section{Discussion}

\section{Main findings}

This study found that the use of a ward initiated multifaceted intervention sustainably reduced the incidence of missed RRS team activations that may result in either unplanned ICU admission or cardiac arrest. This was done without an increase in the number of RRS calls. Taken together these two findings would suggest that the bedside clinical staff can improve the utilization of the RRS. A key finding is that the successful development of co-produced, practice sensitive protocols for initiating
RRS can improve outcomes of patients experiencing deterioration without additional RRS resources.

\section{Study limitations}

There are several significant limitations to this study. The most important limitation of this study is the definition of the term "missed/delayed RRS activation." Technically a missed RRS call should include all patient events where the activation criteria are broached and no RRS call is made at that time. However, to accurately determine this there would need to be either intense manual 
Table 2 Rates of clinical incidents pre- and post- intervention

\begin{tabular}{|c|c|c|c|c|c|c|c|}
\hline & Ward 1 & Ward 2 & Ward 3 & Ward 4 & Ward 5 & Ward 6 & Total \\
\hline \multicolumn{8}{|l|}{ Bed days } \\
\hline Pre-intervention & 1226 & 2033 & 1021 & 3720 & 2166 & 1235 & 11401 \\
\hline Post-intervention & 1321 & 1831 & 1101 & 3100 & 2415 & 2079 & 11847 \\
\hline \multicolumn{8}{|l|}{ RRS activations } \\
\hline \multicolumn{8}{|c|}{ (per 1000 bed days) } \\
\hline Pre-intervention & $16(13.10)$ & $14(6.89)$ & $3(2.94)$ & $33(8.87)$ & $17(7.85)$ & $8(6.48)$ & $91(7.98)$ \\
\hline \multirow[t]{2}{*}{ Post-intervention } & $18(13.63)$ & $19(10.38)$ & $6(5.45)$ & $22(7.10)$ & $20(8.28)$ & $8(3.85)$ & $93(7.85)$ \\
\hline & $(p=0.899)$ & $(p=0.239)$ & $(p=0.381)$ & $(p=0.416)$ & $(p=0.870)$ & $(p=0.296)$ & $(p=0.910)$ \\
\hline \multicolumn{8}{|l|}{ Cardiac Arrests } \\
\hline \multicolumn{8}{|c|}{ (per 1000 bed days) } \\
\hline Pre-intervention & $3(2.45)$ & $2(0.98)$ & $0(0)$ & $4(1.08)$ & $1(0.46)$ & $0(0)$ & $10(0.88)$ \\
\hline \multirow[t]{2}{*}{ Post-intervention } & $2(1.51)$ & $5(2.73)$ & $0(0)$ & $0(0)$ & $0(0)$ & $0(0)$ & $7(0.59)$ \\
\hline & $(p=0.599)$ & $(p=0.222)$ & - & $(p=0.984)$ & $(p=0.987)$ & - & $(p=0.423)$ \\
\hline \multicolumn{8}{|c|}{ Missed RRS activations } \\
\hline \multicolumn{8}{|c|}{ (per 1000 bed days) } \\
\hline Pre-intervention & $3(2.45)$ & $2(0.98)$ & $0(0)$ & $4(1.08)$ & $3(1.39)$ & $0(0)$ & $12(1.05)$ \\
\hline \multirow[t]{2}{*}{ Post-intervention } & $1(0.76)$ & $0(0)$ & $0(0)$ & $1(0.32)$ & $1(0.41)$ & $1(0.48)$ & $4(0.34)$ \\
\hline & $(p=0.309)$ & $(p=0.988)$ & - & $(p=0.281)$ & $(p=0.295)$ & $(p=0.991)$ & $(p=0.049)^{*}$ \\
\hline \multicolumn{8}{|c|}{ Unplanned ICU admissions } \\
\hline \multicolumn{8}{|c|}{ (per 1000 bed days) } \\
\hline Pre-intervention & $6(4.89)$ & $5(2.46)$ & - & - & - & $1(0.81)$ & $12(2.67)$ \\
\hline \multirow[t]{2}{*}{ Post-intervention } & $5(3.79)$ & $2(1.09)$ & - & $5(1.61)$ & $7(2.90)$ & $0(0)$ & $19(1.77)$ \\
\hline & $(p=0.773)$ & $(p=0.444)$ & - & - & - & $(p=0.987)$ & $(p=0.264)$ \\
\hline
\end{tabular}

* = statistically significant.

observation of all written observation and clinical criteria or an automated electronic observation capture system in place. In the first scenario, intense manual observation is likely to lead to skewing of findings by Hawthorne effect and, in the second scenario, observation surveillance by electronic observation capture systems are as yet not widely utilised in the general ward setting. Our previous study found using a snap shot methodology that $42 \%$ of all potential RRS activations were in fact not made $[10,11]$. However, the importance of this is unclear, when the positive and negative predictive value of the various activation criteria, are so low [12-15,23]. However, from a more practical standpoint, our organisation was principally concerned with the missed/delayed RRS activation that was associated with patient harm to such a degree that either intensive care admission was required or, worse, that a cardiac arrest occurred. As such, what mattered to us was not so much that every potential RRS activation was made, but that a culture existed on the wards to positively encourage RRS activation when the patients were clearly deteriorating.

There weresome other limitations to this study. First, that this was a small study undertaken only in six wards in a single organization. The ability to generalize these findings across our organization and in other organizations has not been tested. Second, we do not know if these changes will be sustained over time. We only did an analysis of effectiveness twelve months after the intervention had been implemented. Third, the incidence of missed RRS both pre and post implementation was less than we documented in our baseline point prevalence study. This was due to the different definitions of "missed RRS call" that were used and the different methodology for collecting this data. Fourth, it is possible that the changes we observed between the two data collection periods may have occurred due to a change in case mix of the hospital population. However during this study period there was no major change in the service delivery profile of our hospitals or the population that accessed them.

\section{Study significance}

The fundamental construct of the RRS is that there needs to be a set of rules based on physiological values that determine the activation and response to certain predefined criteria. These rules have been determined by expert opinion and have not been validated [12]. 
Attempts to validate such rules have demonstrated that, on the whole, the activation criteria have reasonable negative predictive value. However, the adherence to rules invariably has a poor positive predictive value generally due to low sensitivity of the criteria [12-15]. For the clinician faced with using the RRS this translates to a set of rules that mean the RRS must be called, but the net result of that is that there is minimal to no intervention and the patient does just fine sitting there in their ward bed [23]. Junior clinicians repeatedly observe this system with a low 'signal to noise' ratio leading to reluctance to activate the RRS and perception that they can manage these situations. This contrasts to the perspective of the RRS teams, the intensive care teams and units and the hospital administrators. For these groups each missed RRS, regardless of incidence that results in patient harm, is a major catastrophe. There is a perception from these groups that the junior staff members were unaware of the seriousness of the patient's condition or did not adequately record the clinical observations. The usual response from administration to these situations is to develop more policy and procedure to be handed down to the workers in the real world of hospital medicine at the bedside [24]. Additional policy and procedures that conflict with the workflow and reduce efficiency of the bedside clinical staff are most likely to be ignored or at best, are followed inconsistently [24]. This is reflected in our experience, with a small but significant incidence of patients that experienced harm as a result of missed RRS calls remaining persistent. In our view this approach has the risk of reinforcing beliefs amongst bedside health care clinicians that they are perceived as incapable and can't manage their patients. Invariably the staff does understand what is happening with their patients who are clinically deteriorating. We found in that study [10] that it was other cultural factors that were at play that prevented appropriate and timely clinical interventions for such patients. By taking the problem to the actual staff on the ground to get them to solve their own problems with their own resources, we succeeded in our aim of the ward teams taking ownership of the problem of deteriorating patients.

\section{Conclusion}

We have described and demonstrated the benefits of a bottom up intervention approach to a significant patient problem in our organization. The use of this innovative way to harness clinical expertise at the ward bedside level within certain predefined boundaries has in our view improved the utilization of the RRS in our organization.

\section{Competing interests}

The authors declare that they have no competing interests.

\section{Authors' contributions}

$\mathrm{MB}, \mathrm{SM}$, and BS all contributed equally to study design and manuscript preparation. MF, TH and TS all undertook data collection and contributed to the study design. SW contributed to the study design. All authors read and approved the final manuscript.

\section{Acknowledgement}

This study was supported by a grant from the Victorian Managed Insurance Authority.

\section{Author details}

${ }^{1}$ The Centre for health Services Research, School of Medicine University of Tasmania, Private Bag 3513, Burnie, Tasmania 7320, Australia. ${ }^{2}$ Monash Injury Research Institute, Monash University and Monash Simulation, Monash Health, Melbourne, Australia. ${ }^{3}$ The Quality Program, Monash Health, Melbourne, Australia

Received: 12 December 2014 Accepted: 21 March 2015 Published: 8 July 2015

References

1. Jones DA, DeVita MA, Bellomo R. Rapid Response Systems. N Engl J Med. 2011;365(2):39-46.

2. MERIT study investigators. Introduction of the medical emergency team (MET) system: a cluster randomised controlled study. Lancet. 2005;365:2091-7.

3. Chen J, Bellomo R, Flabouris A, Hillman K, Finfer S. The relationship between early emergency team calls and serious adverse events. Crit Care Med. 2009;37(1):148-53

4. Hillman KM, Bristow PJ, Chey T, Daffurn K, Jacques T, Norman SL, et al. Antecedents to hospital deaths Intern Med J. 2001;31(6):343-8.

5. Schein RMH, Hazdat N, Pena M, Ruben BH, Sprung CL. Clinical antecedents to in-hospital-cardiopulmonary arrest. Chest. 1990:98(6):1388-92.

6. Franklin C, Mathew J. Developing strategies to prevent in-hospital cardiac arrest: analyzing responses of physicians and nurses in the hours before the event. Crit Care Med. 1994:22:244-7.

7. Smith AF, Wood J. Can some in-hospital cardio-respiratory arrests be prevented? A prospective survey. Resuscitation. 1998;37(3):133-7.

8. Buist MD, Jarmolowski E, Burton PR, Bernard SA, Waxman BP, Anderson J. Recognising clinical instability in hospital patients before cardiac arrest or unplanned admission to Intensive Care. A pilot study in a tertiary-care hospital. Med J Aust. 1999;171:22-5.

9. McQullian P, Pilkington S, Allan A, Taylor B, Short A, Morgan G, et al Confidential inquiry into quality of care before admission to intensive care. BMJ 1998;316(7148):1853-1858.

10. Shearer B, Marshall S, Buist MD, Finnigan M, Kitto S, Hore T, et al. What stops hospital clinical staff from following protocols? An analysis of the incidence and factors behind the failure of bedside clinical staff to activate the rapid response system in a multi-campus Australian metropolitan healthcare service. BMJ Qual Saf. 2012;21(7):569-75.

11. Marshall SD, Kitto S, Shearer W, Wilson SJ, Finnigan MA, Sturgess T, et al. Why don't hospital staff activate the rapid response system? How frequently is it needed and can the process be improved? Implement Sci. 2011;6(1):39-45.

12. Cuthbertson $\mathrm{BH}$, Smith GB. A warning on early- warning scores. Br J Anaesth. 2007:98:704-6.

13. Edwards ED, Mason BW, Oliver A, Powell CV. Cohort study to test the predictability of the Melbourne criteria for the activation of the medical emergency team. Arch Dis Child. 2011;96:174-9.

14. Edwards ED, Powell CV, Mason BW, Oliver A. Prospective cohort study to test the predictability of the Cardiff and Vale paediatric early warning system. Arch Dis Child, 2009;94(8):602-6.

15. Tibballs J, Kinney S. Reduction of hospital mortality and of preventable cardiac arrest and death on introduction of a paediatric medical emergency team. Pediatr Crit Care Med. 2009;10:306-12.

16. Buist MD, Moore GE, Bernard SA, Waxman BP, Anderson JN, Nguyen TV. Effects of a medical emergency team on reduction of incidence of and mortality from unexpected cardiac arrests in hospital: preliminary study. BMJ 2002;324(7334):387-90. 
17. Buist M, Harrison J, Abaloz E, Van Dyk S. Six year audit of cardiac arrests and medical emergency team calls in an outer metropolitan teaching hospital. BMJ. 2007;335(7631):1210-2. Dec 8.

18. Harrison J, Buist M, Flanagan B. A simulation based MET training course for Physician Trainees at Southern health. Melbourne: Abstract book, the 9th National forum for pre-vocational medical education; 2004. October 24-26 $6^{\text {th }}$.

19. National Standards and Accreditation. www.safetyandquality.gov.au/.

20. Marshall SD, Harrison J, Flanagan B. The teaching of a structured tool improves the clarity and content of inter-professional clinical communication. Qual Saf Health Care. 2009;18:137-40.

21. Finnigan M, Marshall SD, Flanagan B. ISBAR for clear communication: one hospital's experience spreading the message. Aust Health Rev. 2010;34:400-4.

22. Chrimes N, Anderson J, Owen D, Jarvis S. VITALS - Vigilant Team Assessment Learning Session for Health Professionals. Adelaide, South Australia: 18th National Prevocational Medical Education Forum; 2013. 3-6th November.

23. Buist M, Nguyen T, Moore G, Bernard S, Anderson J. Association between clinically abnormal bedside observations and subsequent in- hospital mortality: a prospective study. Resuscitation. 2004;62:137-41.

24. Gurses AP, Seidl KL, Vaidya V, Bochicchio G, Harris AD, Hebden J. et al. Systems ambiguity and guideline compliance: a qualitative study of how intensive care units follow evidence-based guidelines to reduce healthcare-associated infections. Qual Saf Health Care. 2008;17(5):351-9.

doi:10.1186/2056-5917-1-8

Cite this article as: Buist et al: Getting more efficient Rapid Response System (RRS) utilization by the use of a general ward based deteriorating patient contract. Safety in Health 2015 1:8.

\section{Submit your next manuscript to BioMed Central and take full advantage of:}

- Convenient online submission

- Thorough peer review

- No space constraints or color figure charges

- Immediate publication on acceptance

- Inclusion in PubMed, CAS, Scopus and Google Scholar

- Research which is freely available for redistribution 